\title{
Importancia de las cartas suicidas en la investigación forense
}

\section{Importance of the suicidal letters in the forensic investigation}

\section{Resumen}

La pericia médico forense tiene básicamente una finalidad probatoria. Resulta importante destacar que la intervención de la medicina forense revele algunos indicios o mensajes referentes y consustanciales al individuo lo que puede ser de ayuda para llegar a establecer el perfil del suicida, sus conflictos y su dinámica.

El análisis y la valoración de las cartas y notas dejadas por aquellos que consuman el suicidio es un componente muy importante que nos permite conocer y analizar los rasgos más significativos y característicos de un individuo. De esta forma se puede establecer el origen, gravedad, evolución y efectos del malestar psíquico del que era portador y que le llevó a realizar dicho acto.

Palabras clave: Notas suicidas. Medicina forense. Suicidio consumado.

\section{Abstract}

The know-how forensic expertise has basically an evidential purpose. It is important to underline that the intervention of the forensic medicine may reveal some indications or relating and consubstantial messages to the individual, which could be used as a help to establish the profile of the suicide, its conflicts and its dynamics. The analysis and evaluation of the letters and notes left by those that consummate the suicide is a very important component that allows us to know and to analyse the most significant features characteristics of an individual. By this way the origin, gravity, evolution and effects of the psychic discomfort he or she was carrying leading to perform the above mentioned act might be established.

Key words: Suicidal notes. Forensic medicine. Consummated suicide.

\section{Introducción}

El suicidio es uno de los temas más complejos y misteriosos a los que se enfrenta el mundo médico y por ello su investigación resulta potencialmente una de las cuestiones más intrigantes para los profesionales, no sólo de la psiquiatría sino también de la medicina forense. Si tratamos de estudiar un determinado suicidio, una de las grandes dificultades que nos encontramos es la obtención de datos que nos permitan componer la situación que llevó a una determinada persona a acabar con su vida.
El estudio del suicidio por parte del médico forense abarca dos aspectos que deben estar ligados en la investigación de la muerte suicida. Estos son, el diagnóstico y las circunstancias de la muerte y la investigación de los motivos o ideación suicida ${ }^{1}$.

De ahí que cuando una persona llega a consumar un acto suicida, la intervención de la medicina forense no debiera quedar únicamente en la determinación de las causas de la muerte, mediante la realización de la autopsia médico legal, sino que además debería resultar interesante que el perito forense, con su preparación, lograra revelar algunos indicios o

\section{Casado Blanco ${ }^{1}$ \\ P. Mata Ron ${ }^{2}$ \\ A. Raya Isla ${ }^{3}$}

${ }^{1}$ Doctor en Medicina. Especialista en Medicina Legal y Forense.

${ }^{2}$ Especialista en Medicina Legal y Forense.

${ }^{3}$ Médico Forense. Instituto de Medicina Legal de Badajoz.

Correspondencia:

Mariano Casado Blanco Instituto de Medicina Legal de Badajoz Campus Universitario Avda. de Elvas s/n

06071 Badajoz

E-mail:

mariano.casado@justicia.es

Fecha de recepción: 22.JUN.2012

Fecha de aceptación: 12.NOV.2012 
mensajes referentes y consustanciales al individuo, que pudieran servir de ayuda para llegar a establecer el perfil del suicida, sus conflictos y su dinámica; de manera que permitiera el reconstruir su personalidad y su estado de salud mental, en momentos previos a cometer el acto suicida.

Todo suicidio consumado deja vestigios en el lugar en que se produce, de ahí que el objetivo de la investigación posterior sea interpretar correctamente los hechos, reconstruir lo ocurrido y comprender lo que sucedió.

Pues bien, dentro de este entramado, el análisis de cartas o notas suicidas manuscritas es uno de los métodos más valiosos en el estudio del comportamiento suicida, ya que se puede considerar como un mensaje por el cual el suicida manifiesta su idea, su responsabilidad y su premeditación a la consumación del suicidio y además pretende que sea vista posterior a su muerte. De ahí que la técnica de análisis de su contenido, sea una de las más utilizadas para la comprensión del suicidio ${ }^{2,3}$.

Su estudio resulta fundamental desde el punto vista psiquiátrico, psicológico y médico-forense, al revelar el carácter bio-psico-social que la persona suicida tiene para tomar esta decisión. A pesar de ello, asumimos que la terminología utilizada en los escritos es un medio esencialmente expresivo de su propio pensamiento en el momento que deciden quitarse la vida, pero también consideramos que en la mente del suicida pudieron haber existido otro tipo de pensamientos que no quedaron reflejados en los escritos ni siquiera puestos de manifiesto por otros medios a sus allegados.

A pesar de que el número de suicidas que dejan este tipo de evidencia es mínimo, cuando éste aparece, nos permite introducirnos en la mente de las víctimas momentos previos al acto suicida y ello como cuestión esencial en la investigación suicidológica, donde a través del análisis de las notas o cartas, se expresan directamente los motivos, sentimientos, deseos, pensamientos del suicida, ya que generalmente son escritas instantes antes de cometer el acto, lo cual nos permite tener un acceso más cercano al acto mismo y, por tanto, a su comprensión ${ }^{4,5}$. En general, las notas de los suicidas se caracterizan por expresar aspectos o eventos muy variables tales como hostilidad y autorreproches, así como por dejar frecuentemente instrucciones a los supervivientes ${ }^{6}$.

En cuanto a las razones por las que se dejan notas escritas son complejas, no existiendo ninguna realidad de que estos mensajes sean más incuestionables que otras formas de comunicación, como pudieran ser las advertencias verbales previas.
De esta forma nos planteamos como objetivos el describir y analizar las notas dejadas por un suicida para así tratar de determinar, en lo posible, las características de tipo afectivas, emocionales, cognoscitivas y de actitudes, relacionadas con el momento en que la víctima ideó su auto-destrucción. Una vez planteada la valoración de las notas manuscritas, solicitamos la oportuna autorización de la autoridad judicial, la cual nos fue concedida manteniendo los aspectos éticos y legales oportunos.

Como método de valoración, aplicamos el esquema de los elementos descritos por Scoott ${ }^{7}$ : causa, modo, motivo, intención, letalidad y suicidio sano frente a demente, destinados a un caso concreto de suicidio consumado, teniendo en cuenta que aunque su aplicación es más específica para la realización de autopsias psicológicas podría resultar útil su utilización para el estudio del caso que exponemos.

\section{Descripción del caso}

Describimos el caso de una mujer, de mediana edad, con una vida estable tanto desde el punto de vista familiar como laboral, que apareció cadáver sobre la cama, en posición decúbito supino, semicubierta con una sábana y con una data estimada en torno a las treinta y seis horas. Como señales externas de violencia, se determinó la presencia de una pequeña puntura a nivel del flanco derecho.

En la inspección del lugar de los hechos, se encontró un vaso sin contenido y un sobre dirigido a su familia. Dentro del sobre había una caja del fármaco "Ioracepam" de $1 \mathrm{mg}$, en el que se contabilizaron la falta de catorce comprimidos, así como una carta manuscrita de catorce folios, en la que explicaba sus sentimientos, su problemática emocional y donde igualmente especificaba una serie de recomendaciones acerca de determinados aspectos de su vida (cuentas bancarias, reparto de joyas y objetos personales, instrucciones para el trabajo...).

Siguiendo con la inspección ocular, en el cuarto de baño, se encontraron 5 dispensadores de insulina así como 5 viales, todos ellos sin contenido y junto a estos una receta de "humalog" e instrucciones para su administración.

Aplicando el esquema anteriormente indicado ${ }^{7}$, nos encontramos con:

1. La causa, es la que nos explica cómo murió la persona y si existe una relación entre los hallazgos autópsicos y la muerte, así como si el probable consumo de sustancias (insulina y psicofármacos) 
pudiera tener relación con la muerte. A este respecto, el informe médico forense es el encargado de aclarar la causa de la muerte. El estudio químico-toxicológico determinó la existencia de una sobredosis de insulina.

Para establecer si la insulinemia estaba elevada como consecuencia de una sobredosis exógena, se solicitó la determinación de la glucorraquia y del péptido $C$ (péptido sintetizado en el páncreas unido a la insulina endógena y que se libera parcialmente al torrente sanguíneo), ya que las insulinas humanas exógenas comercialmente disponibles no contienen fragmentos de péptido $C$. Los resultados analíticos fueron de glucosa en LCR de $1 \mathrm{mg} / \mathrm{dl}$ (40-70 mg/dl valores de referencia en ayunas) y de $0,61 \mathrm{ng} / \mathrm{ml}$ de péptido $C$ en suero (1,1-5 valores de referencia). Estos resultados llevaron a determinar la causa de la muerte como una intoxicación medicamentosa.

Analizando someramente el mecanismo fisiopatológico, se puede indicar que la concentración de la glucosa tiene un papel principal en la regulación de la liberación de insulina y ésta se une a un receptor específico tipo proteína-cinasa localizada en la superficie celular, particularmente a nivel del hígado, músculo y tejido adiposo.

In vivo la insulina se sintetiza en las células beta del páncreas como una pro-hormona que al ser fragmentada resulta en el péptido $\mathrm{C}$ y la insulina misma. Los sitios principales de degradación de la insulina son el hígado (70\%) y los riñones (10-40\%). Después de la administración intravenosa o subcutánea de insulina, la vida media será dependiente de la dosis y del tipo de insulina.

Las concentraciones suprafisiológicas condicionan una mayor duración del efecto hipoglucemiante, probablemente por saturación de los sistemas hepáticos de depuración.

2. Otra cuestión, que además puede resultar más ambigua es el modo, hace referencia a las circunstancias que dan lugar a la causa de la muerte. Para ello es preciso recurrir al clásico esquema de muerte natural, accidental, suicida u homicida. Generalmente tanto la causa como el modo de la muerte pueden establecerse a partir de evidencias objetivas que se recopilan en el momento del levantamiento del cadáver, mediante la búsqueda de indicios que nos pudieran revelar las circunstancias de la muerte. Es un apartado esencial, pues éste determinará la necesidad de iniciar o proseguir con la investigación judicial. En el caso que presentamos las propias evidencias nos orientan por un suicidio, sin embargo, en otras ocasiones no resulta fácil determinar el modo en que muere una persona, con base en la información disponible. De esta forma, podemos referenciar determinadas dudas que se generan entre algunos accidentes y suicidios.

3. Una vez determinado que el modo es suicida, el siguiente paso es establecer el motivo por el que la víctima llegó a consumar el suicidio. En muchas situaciones, el motivo es totalmente desconocido y se debe deducir a partir de las evidencias disponibles.

Para ello y siguiendo a Shneidman ${ }^{5}$ recomienda una revisión cuidadosa de tres cuestiones: ¿por qué la víctima hizo esto?, ¿cuáles fueron sus razones? y ¿por qué lo hizo en ese momento determinado?

En estas situaciones es elemental la información sobre la vida, comportamiento y condiciones psicológicas de la suicida, de forma que la investigación pueda evaluar cuál pudiera haber sido el motivo más probable.

Para ello resulta interesante acudir a la valoración de los antecedentes clínicos de la víctima determinados en los informes clínicos. En éstos podemos determinar cómo desde hacía más de veinte años presentaba evaluaciones diagnósticas retrospectivas según los criterios de DSM consistentes en: Eje I: trastorno depresivo mayor. Eje II: trastorno de personalidad mixto. Eje III: no enfermedades médicas y Eje IV: buen apoyo familiar. Con respecto a estos diagnósticos, es evidente que la depresión mayor es el trastorno mental individual que más frecuentemente precede al acto suicida y por ello el más relacionado con el riesgo suicida ${ }^{8-12}$.

En el caso, se contabilizan varios ingresos psiquiátricos involuntarios urgentes, todos ellos relacionados con intentos de autolisis, para los cuales utilizó diferentes métodos más o menos cruentos como ingesta masiva de psicofármacos (antidepresivos tricíclicos), ingesta de quetiapina (neuroléptico antipsicótico atípico), utilización de cúter e ingesta masiva de paracetamol.

Tomando como referencia estos antecedentes clínicos y sobre todo del análisis del manuscrito dejado, nos pueden dar respuesta a las cuestiones indicadas y ello considerando que la escritura también es base de la personalidad. La manera en cómo escribe, la forma y tamaño de las letras son algunas de las características que permiten conocer cómo era nuestra víctima y su grado de desesperanza.

Si damos una visión psicoanalítica al caso, nos revela que la suicida era una persona con una capacidad de creación y producción disminuidas y, a veces, anuladas. Su vivencia subjetiva correspondía a la 
anulación de todo deseo y de todo placer que llegaba a afectar hasta los mínimos actos de la vida diaria en todos sus aspectos (familiar, laboral, social...). De su relato se puede entrever la existencia de un estado que invadía tanto el cuerpo como el pensamiento, acompañado de un sentimiento de desesperación, puesto de manifiesto en una experiencia de absoluta aniquilación de sí misma, con una marcada ideación activa.

De todo ello y valorando los manuscritos se pueden extraer las siguientes consideraciones:

- Que la víctima presentaba una consideración negativa de sí misma, mostrando una marcada tendencia a considerarse como deficiente, inadecuada e inútil y atribuyendo sus experiencias desagradables a defectos morales o mentales: "he dejado de ser yo", "estoy cansada de experimentos", "estoy trastornada... lo malo es que me doy cuenta y sufro", "estoy muy chalada, mucho...".

- Manifestaba una tendencia a rechazarse a sí misma, creyendo que los demás la rechazaban: "no me siento entendida", "te vas viendo cada día peor... diluyéndote, perdiéndote", "dejadme descansar, o salir de esto que me ata".

- Presentaba una consideración negativa del mundo: "Ios médicos hablan de lo que ellos creen, pero de lo que no saben debe haber mucho porque sino yo no estaría así. La mente es tan oscura y se sabe tan poco de ella", "que dejen de experimentar conmigo".

- Se sentía derrotada socialmente: "estoy cansada de luchar y luchar, del sufrimiento y del no disfrutar de la vida", "a estas alturas me da igual ya lo que piensen de mí".

- Consideraba al mundo como muy exigente y presentándole obstáculos que se interferían con el logro de los objetivos de su vida: "esa ansiedad me ha devorado por dentro", "no hay mal que 100 años dure ni cuerpo que lo resista, yo ya no resisto más", "no puedo más y sólo Dios sabe los esfuerzos que tengo que hacer todos los días para que no notéis mi dolor".

- Tenía una consideración negativa del futuro. Veía el futuro desde una perspectiva negativa y le daba vueltas y más vueltas a una serie de expectativas negativas: "aunque digan que tengo recursos, no es cierto... y necesito ayuda, pero lo que hay hoy día no me sirve".

- Preveía que sus problemas y experiencias comunes continuarían indefinidamente y que se le amontonarían otras muchas peores en su vida: "la esperanza es lo último que se pierde, pero se pierde, aunque sea lo último y yo la perdí el día aquel que me descoloqué".

- Presentaba una tendencia a deformar sus experiencias, malinterpretar acontecimientos concretos e irrelevantes tomándolos como fracasos, privaciones o rechazo personal: "sólo yo sé 10 que he luchado y por lo que he pasado. Sólo yo, los demás veis los toros desde la barrera", "además esto me ha hecho mala persona. Por eso no disfruto de la vida".

- Todo parecía siempre que iba en contra de sí misma, acentuando lo negativo hasta llegar a excluir los hechos positivos: "tú dices que te destrozo la vida, la mía está ya destrozada. No quiero amigos, trabajo, hablar", "nunca fui una persona pesimista, ni amargada y ahora lo soy, por eso sé que no soy yo".

4. El siguiente paso es determinar la intención, la cual representa la resolución de un individuo de llevar a cabo su muerte de forma consciente o inconsciente. En este apartado, es preciso analizar u objetivizar si la suicida había manifestado o comunicado su intención y si éstas llegaron a ser verbalizadas $y / 0$ expresadas repetidamente a personas diferentes o por el contrario la expresión de la intención suicida fue de inicio reciente y atípico.

La comunicación de las intenciones que presenta un suicida hay que considerarlas como un signo evidente de riesgo de suicidio. Existen muchas maneras de efectuar esta comunicación bien a los propios familiares o allegados o incluso a los propios profesionales (médicos, psiquiatras, psicólogos...).

De la revisión de la documentación clínica se puede afirmar que la víctima tenía una intención decidida de acabar con su vida, como lo demuestra el hecho de haber llevado a cabo cinco intentos previos e incluso el último en fechas recientes a la consumación del acto suicida, lo que relaciona un mayor grado de planificación del acto suicida en los cuadros depresivos $^{13}$. En su escrito se nos revelan estos datos cuando manifiesta: "todos los tratamientos los he rechazado (y que sigo haciéndolo pues sino estaría mucho mejor) son muy importantes y nadie les ha hecho ni puto caso", "ipor qué os creéis que os pregunto tanto si me veis mejor?", "ahora no son impulsos, es algo planeado".

5. Una vez determinada la intención efectuamos una valoración del grado de letalidad del método utilizado. El grado de letalidad puede variar y ser alto, medio, bajo o ausente. Como ejemplos podemos indicar que se trata de una letalidad alta cuando existe 
una decisión inequívoca de quitarse la vida (disparo en la cabeza o precipitarse desde una gran altura).

La letalidad será media cuando el suicida prepara su propia muerte mediante acciones conscientes 0 inconscientes, como ocurre por ejemplo al descuidar un determinado tratamiento médico que puede salvar su vida o el uso potencialmente letal de drogas o alcohol.

Será letalidad baja cuando la víctima desempeña algún papel pequeño aunque no insignificante, para efectuar o preparar su propia muerte. Y la letalidad estará ausente cuando la víctima no llegue a desempeñar ningún papel para causar su propia muerte, aunque existan datos objetivos con respecto a que el suicida deseaba vivir.

Atendiendo al método utilizado en este caso concreto, sobredosis de insulina, se podría considerar como de letalidad alta y/o media.

6. Y, por último, determinar si una muerte se debió a un suicidio sano frente a demente, lo cual queda estrechamente relacionado con la evaluación de la intención de la persona de quitarse la vida. Un suicidio "sano" indica que la persona tenía comprensión racional de que una acción que realizara resultaría en su propia muerte. Por el contrario, un suicidio "demente" ocurre cuando una persona está tan alterada emocionalmente que no aprecia racionalmente la relación de sus acciones para causar su muerte. En el caso que analizamos calificamos el suicidio como "sano", porque se entiende que la suicida aún estando en posesión de sus facultades de razonamiento deseaba acabar de forma intencionada con su vida. Para llegar a esta tipificación entendemos que a pesar de que se determina un estado mental angustiado es evidente que la suicida había llevado a cabo acciones que muestran una suficiente capacidad para comprender la naturaleza del acto que iba a realizar y sus consecuencias, con la exclusiva finalidad de atentar contra su propia vida.

\section{Conclusiones}

Aunque el análisis y valoración de las cartas y notas suicidas no es una práctica habitual, que sea solicitada por parte del sistema judicial, consideramos que bien puede servir como complemento al trabajo pericial investigador del médico forense, de forma que su aplicación concreta debe servir para realizar el análisis de las circunstancias de muerte de una persona.

Encontrar notas cerca o junto al suicida debe considerarse como una cuestión de gran importancia, ya que brinda información sobre el estado psicológico del mismo, momentos antes de consumar el suicidio. El análisis de estos escritos nos pueden determinar el grado de gravedad del proceso psicopatológico, determinar el grado de impulsividad, los niveles de desesperanza, los problemas vitales, las estrategias de afrontamiento del paciente incluso el grado de soporte social y familiar, ya que el paso a la acción debe estar en íntima relación con el grado de desesperación del suicida.

Del mismo modo, de la valoración de los escritos se puede igualmente determinar la intencionalidad letal que pudiera tener. Así, en los casos, en que se determina que el acto suicida ha sido llevado a cabo con premeditación y planificación, nos orienta por un paciente portador de un trastorno depresivo, muy diferente de aquellos donde el proceso patológico es un trastorno de personalidad, trastorno adaptativo o incluso una toxicomanía, más inclinados a la realización de actos impulsivos.

\section{Bibliografía}

1. Jiménez Féliz J, García Caballero A. Características forenses, psicológicas y lingüísticas de una muestra de notas suicidas en Galicia. Boletín Galego de Medicina Legal e Forense 2010;17:31-47.

2. Leenaars A. Suicide notes. New Cork: Human Sciencies Press, 1992.

3. Leenaars A, Haines J, Wenckstern S, Williams C Lester D. Suicide notes from Australia and the

United States. Perceptual and Motor Skills 2003;92:1281-2.

4. Leenaars A, DeWilde E, Wencksern S, Oral M. Suicides notes of adolescents: a life-span comparison. Canadian Journal of Behavioral Science, 2001;33:47-57.

5. Shneidman E. Autopsy of a suicidal mind. New Cork: Oxford University Press, 2004.

6. Sarró B, De La Cruz C. Los suicidios. Barcelona: Ed. Martínez Roca. 1991. 
7. Scoott ChL, Swartz E, Warburton K. Autopsia psicológica: resolver los misterios de la muerte. Psychiatr Clín N Am 2006;29:805-22.

8. Conwell $\mathrm{Y}$, Duberstein P, Cox C, Herrmann JH, Forbes NT, Caine ED. Relationships of age- and axis I diagnoses in victims of completed suicide: a psychological autopsy study. Am J Psychiatry 1996;153:1001-8.

9. Appleby L, Cooper J, Amos T, Faragher B. Psychological autopsy of suicides by people under. $\mathrm{Br} \mathrm{J}$ Psychiatry 1999;175:168-74.

10. Henriksson MM, Aro HM Marttunen MJ, Heikkinen $\mathrm{ME}$, Isometsä ET, Kuoppasalmi KI, et al. Mental disorders and comorbidity in suicide. Am J Psychiatry 1993;150:935-40.

11. Lesage AD, Boyer R, Grunberg F, Vanier C, Morissette $R$, Ménard-Buteau $C$, et al. Suicide and mental disorders: a case-control study of young men. Am J Psychiatry 1994;151:1063-8.

12. Casado Blanco M. Suicidios en mayores de 65 años en la provincia de Badajoz: análisis médico legal. Cuad. Med. Forense. 2002;28:25-32.

13. Portilla Arroyo Jl. Evaluación del riesgo de suicidio en los servicios de urgencias. En: Bobes J, Sáiz martínez PA, García-Portilla González, et al. Comportamientos Suicidas. Prevención y Tratamiento. Barcelona: Ars Médica. 2004 